\title{
Fluid Management in Septic Shock: a Review of Physiology, Goal-Directed Therapy, Fluid Dose, and Selection
}

\author{
Michael W. Best ${ }^{1} \cdot$ Craig S. Jabaley ${ }^{1,2}$ \\ Published online: 22 April 2019 \\ (C) Springer Science+Business Media, LLC, part of Springer Nature 2019
}

\begin{abstract}
Purpose of Review To review our current understanding of the intravascular space, therapeutic goals of fluid resuscitation, suitable endpoints of resuscitation, and appropriate choice of fluids.

Recent Findings An evolving understanding of the endothelium and glycocalyx has improved our understanding of the intravascular space. More aggressive fluid resuscitation after adoption of the Surviving Sepsis Campaign guidelines has led to an initial reduction in mortality associated with sepsis; however, the untoward effects of volume overload are increasingly evident. Fluid responsiveness is likely the best endpoint for resuscitation. Albumin has not been shown to be superior to crystalloids in the resuscitation of septic patients. As $0.9 \%$ saline has been associated with acute kidney injury, balanced buffered salt solutions are considered the preferred resuscitation fluid of choice.

Summary Fluid resuscitation with buffered crystalloid solutions is preferred and should likely continue if the patient remains fluid responsive. This strategy risks volume overload.
\end{abstract}

Keywords Sepsis $\cdot$ Septic shock $\cdot$ Resuscitation $\cdot$ Intravenous fluids $\cdot$ Goal-directed therapy $\cdot$ Crystalloids $\cdot$ Colloids

\section{Introduction}

Septic shock is defined as a dysregulated host response to infection with evidence of organ dysfunction secondary to inadequate perfusion [1]. More broadly, septic shock can be conceptualized as an imbalance between the demand, delivery, and utilization of oxygen at the cellular level due to the interplay between infection and the body's reaction to infection. Maintenance of adequate intravascular volume to promote cardiac output and oxygen delivery during concomitant systemic vasodilation has become a mainstay of practice since the adoption of early goal-directed therapy (EGDT) and the subsequent development of the Surviving Sepsis Campaign Guidelines $[2,3 \bullet \bullet, 4 \bullet \cdot]$. Following the original EGDT trial,

This article is part of the Topical Collection on Critical Care Anesthesia

Michael W. Best

michael.w.best@emory.edu

1 Department of Anesthesiology, Division of Critical Care Medicine, Emory University, 1750 Gambrell Dr, T5L27, Atlanta, GA 30322, USA

2 Anesthesiology Service Line, Division of Critical Care Medicine, Atlanta Veterans Affairs Medical Center, Decatur, GA, USA debate persists about the optimal hemodynamic goals and approach to their restoration; however, the general principles of attempting to optimize oxygen delivery, consumption, and

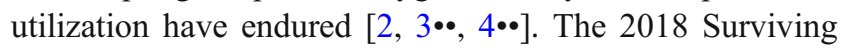
Sepsis Campaign bundle highlights the early administration of antibiotics and an intravenous fluid bolus (IVFB) of $30 \mathrm{~mL} /$ $\mathrm{kg}$ as the cornerstone of its therapeutic recommendations $[4 \bullet \cdot]$. As the heterogenous body of evidence surrounding fluid resuscitation grows in size, scope, and complexity, questions remain concerning both which types of fluids to administer and which physiologic endpoints to use for determining adequate volume resuscitation. This article will review our current understanding of the intravascular space, therapeutic goals of fluid resuscitation, pragmatic fluid selection, and parameters for deciding the appropriate dose thereof.

\section{Revisiting Starling}

Water outside of the cells exists in either the extracellular matrix (ECM) or intravascular space. Starling described how oncotic and hydrostatic pressures acting across the capillary membrane determine the balance of water between the ECM and the intravascular space. Conceptually, hydrostatic 
pressure in excess of oncotic pressure would beget translocation of water from the intravascular space into the ECM. If oncotic pressure dominates, water should flow from the ECM back into the intravascular space. Although Starling's conceptual model has enjoyed great longevity, some of its theoretical tenants have not withstood closer scrutiny [5].

We now understand that a complex, metabolically active, and seemingly fragile glycoprotein and proteoglycan matrix lines the luminal wall of the endothelium, which has been termed the glycocalyx or endothelial glycocalyx layer (EGL) [5-9]. At the level of the capillary, the EGL serves as a semipermeable barrier that prevents the passage of larger molecules (e.g., albumin) through fenestrations and gaps between endothelial cells (Fig. 1). The EGL is largely responsible for establishing the oncotic gradient described in Starling's model. In practice, however, the inward oncotic pressure can only attenuate but not overcome hydrostatic pressure. Except in a limited few tissue beds, water does not cross from the ECM into the intravascular space directly; instead, water in the ECM returns to the intravascular space via the lymphatics.

\section{Intravascular Changes During Sepsis}

Sepsis and its associated widespread inflammatory response create several key changes to the intravascular space and its structure [5, 7-9]. An increase in nitric oxide synthetase and a relative deficiency in vasopressin results in vasodilation in both the arterial and venous beds [9]. The dilation of the highly elastic arteries primarily results in reduced mean arterial pressure; dilation of the highly compliant veins primarily results in increased potential venous capacitance and resultant relative hypovolemia.

Multiple inflammatory markers and endothelial adhesion molecules activated during sepsis cause cellular changes at the endovascular level, which can beget both macrocirculatory and microcirculatory dysfunction through endotheliopathy [10•]. In addition to endothelial injury, microvascular thrombi, and widening of gaps between endothelial cells, these inflammatory mediators have also been linked to the shedding of the EGL. Circulating levels of heparan sulfate, syndecan-1, and other large molecular constituents of the EGL are heightened in states of shock and trauma [8]. The loss of the EGL contributes to several phenomena observed in severe sepsis:

1. Experimental evidence suggests that the EGL can account for 700 to $1500 \mathrm{~mL}$ of intravascular volume $[5,6]$. The loss of the EGL therefore exacerbates the relative hypovolemia from vasodilation described previously.

2. Degradation of the EGL allows molecules to pass through cellular gaps and fenestrations in the endothelial walls. This altered equilibration of macromolecules across the capillary membrane leaves outward hydrostatic pressures unopposed, increasing the rate at which water escapes from the intravascular space to the ECM (i.e., capillary leak). The increased rate of capillary leak overwhelms the lymphatics, and anasarca develops $[5,7,9]$.

3. The release of glycocalyx-bound inflammatory and adhesion molecules exposes endothelial cells and potentiates the inflammatory process $\left[10^{\bullet}\right]$.
Fig. 1 Diagram depicting a healthy (top) and damaged (bottom) endothelial glycocalyx layer on the luminal side of the endothelium. (1) Adhesion molecules, (2)

Glycosaminoglycans, (3) Glycocalyx-bound mediators, (4) Endothelial cells, (5) Endothelial basemen membrane. Reproduced with permission from Santi M, Lava SA, Camozzi P, et al. "The great fluid debate: sale or socalled "balanced" salt solutions?" Ital J Pediatr. 2015 Jun 25; 41:47, doi: https://doi.org/10.1186/ s13052-015-0154-2
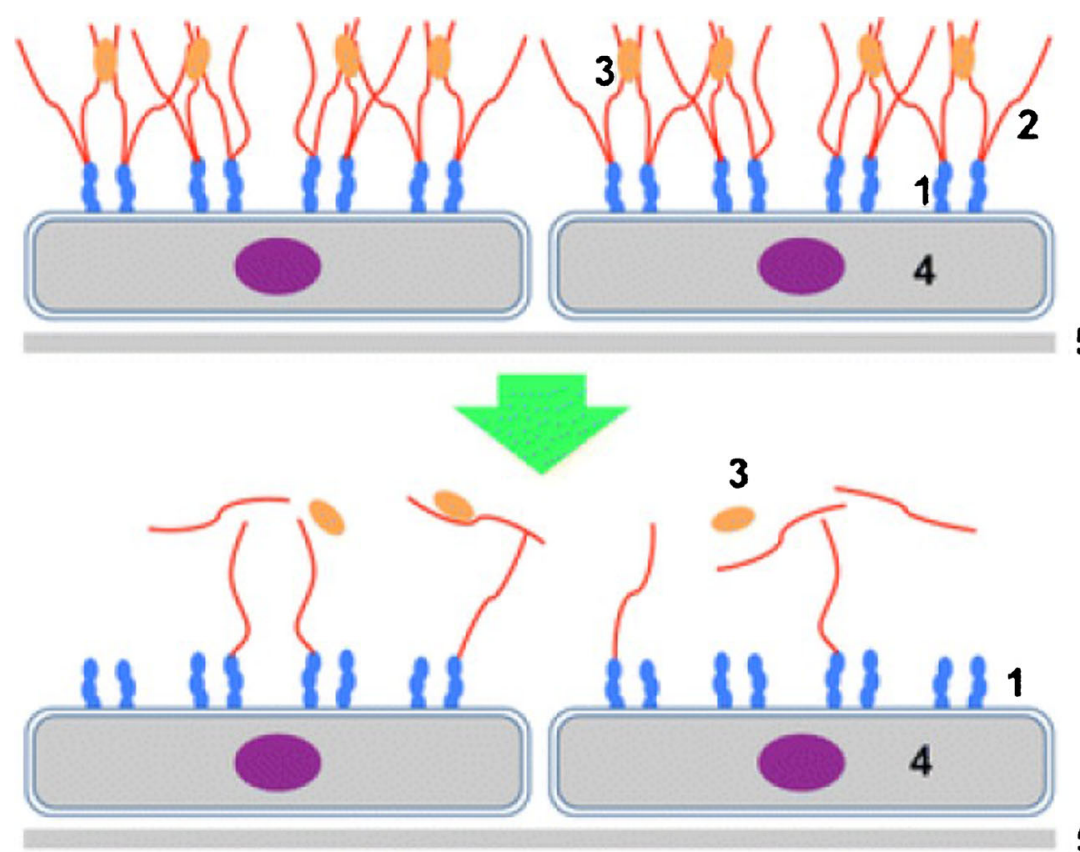


\section{Goals of Fluid Resuscitation During Sepsis}

The overarching goals in fluid resuscitation of the septic patient are to replete the unstressed volume and therefore optimize preload.

Fluid within the venous vasculature can be conceptualized as belonging to either an unstressed or stressed volume [5, 7, 9, $11 \cdot, 12]$. The unstressed volume is the amount of fluid required before intravascular pressure begins to rise, which can also be thought of as the volume prior to the inflection point on the compliance curve. Stressed volume, then, is the amount of fluid that actively contributes to vascular wall stress, venous pressure, and therefore venous return. Splanchnic and cutaneous venous beds are large venous capacitance reservoirs and the primary contributors to the unstressed volume, which can be recruited to maintain preload. Early fluid resuscitation in sepsis initially fills the unstressed volume created by both vasodilation and loss of the EGL. Vasopressors can also affect venous tone, shift unstressed blood out of the capacitance vessels, and contribute to preload. Continued fluid resuscitation then increases the stressed volume, which promotes cardiac output. Once the unstressed volume is replete, fluid resuscitation becomes a balancing act. Too little stressed volume decreases venous return and cardiac output; too much increases outward hydrostatic pressure and capillary leak.

Venous return is a major determinant of ventricular enddiastolic volume and therefore cardiac preload $[5,7,9,11$, 12]. The Frank-Starling curve is defined by the length-tension relationship observed in the myocardium (Fig. 2) [6]. As muscle fibers are stretched, increased actin-myosin cross-bridging results in increased contractility. Therefore, stroke volume initially increases as end-diastolic volume increases. As the myocardium continues to be stretched, there is impairment to actin-myosin cross-bridging and ventricular dysfunction ensues. The slope of the curve is relatively shallow for an otherwise compliant ventricle but steepens with ventricular hypertrophy and diastolic dysfunction. Concomitantly, attention must be directed to filling pressures to ensure that a favorable gradient for venous return is maintained such that venous backpressure does not compromise organ perfusion [7].

The correct amount of fluid for a patient is therefore dependent on vascular and cardiac compliance. The ideal fluid volume would provide enough cardiac preload to function near the apex of the Frank-Starling curve without creating excessive intravascular hydrostatic pressure. It may not be possible to achieve this ideal fluid balance, particularly in patients with poor vascular and/or ventricular compliances. These patients have much narrower therapeutic windows and require closer monitoring and judicious fluid administrations. Fluid shifts and dynamic changes in compliances further challenge the management of these patients.

\section{Endpoints of Resuscitation}

Several clinical variables have been proposed to predict when a patient has an optimized fluid balance [7, 9, 11•, 12-15]. Rivers and colleagues showed that a protocol optimizing

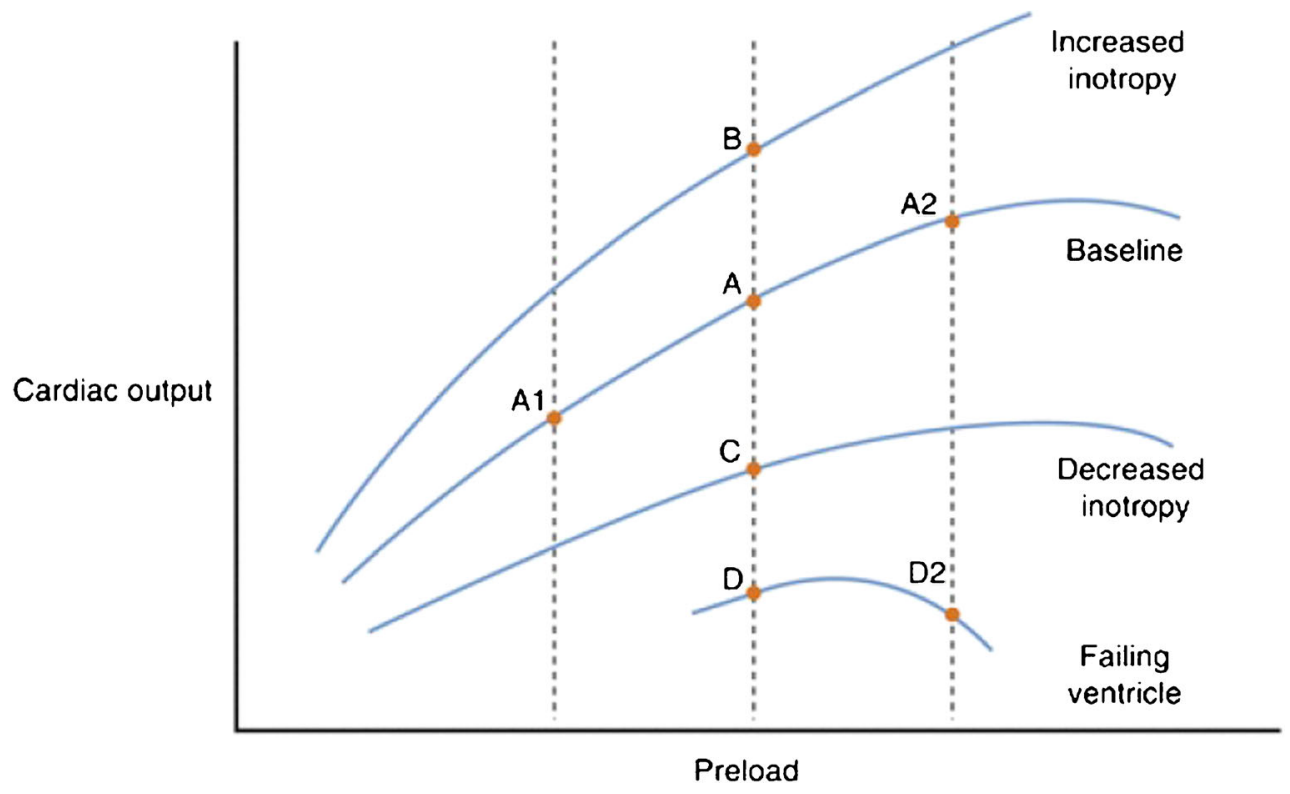

Fig. 2 The Frank-Starling curve demonstrating the relationship between cardiac output and preload. The vertical (dashed) lines demonstrate similar levels of preload and how they relate at various ventricular inotropic states. A1, A, and A2 demonstrate how increasing preload often leads to increased cardiac output along a baseline Frank-Starling curve. D and D2 demonstrate how increasing preload can lead to worsened cardiac output in the failing ventricle. A, B, C, D compare how changing the inotropy of the heart with a fixed preload can result in different cardiac outputs. Reproduced from Mann D. (2017) "FrankStarling Curve.” In: Raj TD (Ed.) Data Interpretation in Anesthesia - A Clinical Guide. Springer, Cham, with permission from Springer Nature 
central venous pressure (CVP), mean arterial pressure (MAP), and oxygen delivery (defined in this trial by central venous oxygen saturation, hemoglobin, cardiac output, and oxygen consumption) significantly reduced mortality associated with sepsis [2]. Termed EGDT, this protocol initially used CVP to guide fluid resuscitation and then vasopressors and inotropes to manipulate the vascular compliance and Frank-Starling curves, respectively. Blood products were also used to increase the oxygen carrying capacity with a targeted hemoglobin of $10 \mathrm{~g} / \mathrm{dL}$.

Although the specific endpoints proposed by Dr. Rivers have come under significant debate, EGDT shifted management beyond simply targeting blood pressure and CVP. Several subsequent studies that used the same or similar protocols as Dr. Rivers' team were unable to demonstrate a mortality benefit [16-21]. Many of these trials occurred after widespread adoption of the Surviving Sepsis Campaign Guidelines, which incorporated several key components of the original EGDT protocol [3••].

Current sepsis guidelines suggest administering an initial IVFB which serves to replete the unstressed volume $[3 \bullet \bullet, 4 \bullet \bullet]$. Fluid resuscitation is then continued as long as the patient is "fluid responsive," defined as an ability to increase cardiac output with additional IVFB $[3 \bullet \bullet, 7,11 \bullet, 12,15]$. The assumption is made that if a patient is fluid responsive, then they are to the left of the apex of the Frank-Starling curve and can benefit from additional venous return.

Several clinical markers have been used to predict if a patient is fluid responsive. One such approach relies on examination of dynamic changes in the macrocirculation during respirophasic variation in intrathoracic pressure during positive pressure ventilation $[11 \bullet 12,15]$. Respiratory variation of pulse pressure (PPV), systolic blood pressure (SPV), and stroke volume (SVV) have all been validated as methods to predict which patients will be fluid responsive provided that a patient is receiving positive pressure ventilation, is compliant with the ventilator, is in a regular cardiac rhythm, and is receiving a tidal volume of at least $8-10 \mathrm{~mL} / \mathrm{kg}$ of predicted body weight. In these patients, variations of greater than 10 $13 \%$ reasonably predict fluid responsiveness. In spontaneously breathing patients, sonographic measurement of the respiratory variation in the diameter of the inferior vena cava has also been shown to reasonably predict fluid responsiveness. These maneuvers are additional pieces of information that must be utilized within the context of the patient's physical exam, clinical course, and underlying pathophysiology.

Once the clinician believes a patient might benefit from additional fluid, it is important for an objective marker to be used to assess whether the additional fluid was beneficial. In modern practice, some combination of stroke volume, cardiac output, MAP, central or mixed venous oxygen saturation, and lactate clearance are commonly evaluated in concert $[3 \bullet \bullet, 7,9$, $11 \cdot, 12,13,15,22]$. If the patient has a suspected narrow therapeutic window such that an empiric fluid challenge is imprudent, then a passive leg raise test can be employed $[11 \cdot 12]$. By placing a patient supine and raising the legs to greater than 45 degrees, approximately $500 \mathrm{~mL}$ of blood volume can be recruited from the venous capacitance vessels. This maneuver examines a patient's response to a fluid bolus without committing to the treatment plan. Once the patient no longer demonstrates a significant response, then vasopressors or inotropes instead should be utilized.

Although markers of fluid responsiveness are highly sensitive in identifying patients who are hypovolemic, they are not specific and risk over-resuscitation [7,9]. It is the authors' belief that the lack of significant difference between EGDT and current best practices suggests that reliable clinical endpoints of resuscitation still have not been elucidated. Most clinical monitors provide information about the macrocirculation-mean arterial pressure, heart rate, cardiac output, etc. Even measures of end-organ function, such as urine output, have not been demonstrated to correlate meaningfully with tissue perfusion [14]. Multiple studies have demonstrated that optimizing the macrocirculation does not guarantee an optimized microcirculation, which represents an important area of future study in conjunction with the exploration of therapeutic interventions targeting the EGL $[23 \cdot, 24]$.

\section{Colloids Versus Crystalloids}

Two broad classes of intravenous (IV) fluids are available: colloids and crystalloids. Three major categories of colloids are commercially available: albumin, hydroxyethyl starch (HES), and gelatin. As oncotically active fluids, colloids hypothetically slow the rate of capillary leak by increasing the inward oncotic pressure across the capillary membrane; however, this effect is short-lived in septic patients as the loss of the EGL will allow oncotically active molecules to ultimately equilibrate between the intravascular and extracellular spaces $[5,7,9]$. Studies of the intravascular half-lives of colloids and crystalloids are consistent, demonstrating a longer intravascular half-life for colloids but eventual capillary leakage into the ECM for both [25]. Other possible benefits of colloids, namely albumin, include largely hypothetical anti-inflammatory effects and nitric oxide-scavenging properties [26].

There have not yet been any large, randomized controlled trials that have demonstrated a clear difference in mortality between resuscitation using crystalloids or colloids in sepsis. The SAFE trial was a large, pragmatic randomized controlled trial that examined $0.9 \%$ saline versus albumin as a resuscitative fluid for a diverse population of critically ill adults [27]. Although no significant difference in 28-day mortality was 
seen for all patients, there was a suggestion of improved outcomes in patients with severe sepsis and acute respiratory distress syndrome (ARDS). Two subsequent studies also demonstrated slight mortality benefits associated with the use of colloids in patients with severe sepsis, although neither achieved statistical significance [26, 28].

The ALBIOS trial examined albumin repletion in patients with severe sepsis and septic shock [26]. Serum albumin was maintained at $3 \mathrm{~g} / \mathrm{dL}$ by administering $20 \%$ albumin. Patients were otherwise resuscitated with crystalloid solutions. Although there was no change in 28-day or 90-day mortality, post-hoc analysis suggested a possible mortality benefit for those patients in septic shock, although this conclusion was subjected to significant post-publication scrutiny [29].

While administration of albumin for fluid resuscitation has not been convincingly associated with improved clinical outcomes in critically ill adults, a sub-group analysis of the SAFE trial data demonstrated worsened outcomes for patients with severe traumatic brain injury (TBI) who were resuscitated with albumin [30].

HES solutions have been linked to acute kidney injury (AKI) in critically ill patients with significant subsequent regulatory scrutiny by the Food and Drug Administration in the US and the European Medicines Agency in the EU [31-33]. Their subsequent worldwide utilization has markedly declined [15]. The largest prospective study including HES in critically ill adults was the CRISTAL trial: a multi-continent, open-label study that compared outcomes of 2857 patients admitted to the intensive care unit (ICU) [28]. Although there was no difference in 28day mortality, there was a statistically significant decrease in 90-day mortality for those resuscitated with colloids (RR 0.92, 95\% CI 0.86-0.99). The colloid group was also shown to have lower vasopressor requirements and decreased ventilator days. This trial did not differentiate between hypertonic and isotonic crystalloid solutions. It also included HES and other colloid formulations no longer in common use for critically ill patients.

Another important class of colloids are blood products. The TRICC trial initially demonstrated that a lower hemoglobin target of 7-9 g/dL was safe in critically ill patients [34]. A similar trial that considered only patients in septic shock, the TRISS trial, compared hemoglobin goals of $7 \mathrm{~g} / \mathrm{dL}$ versus $9 \mathrm{~g} / \mathrm{dL}$ [35]. There was no difference in 90-day mortality between the groups, although the lower hemoglobin goal of $7 \mathrm{~g} / \mathrm{dL}$ was associated with fewer transfusions (an average of 1 unit compared to 3 units). Given the associated risks, costs, and limited supply of blood products, current best practices recommend blood products in resuscitation only when correcting an anemia or coagulopathy [3••]. Higher hemoglobin goals may be appropriate based on a patient's comorbidities and the overall clinical scenario.
The most recent Surviving Sepsis Campaign Guidelines recommend against the use of colloids as an initial resuscitative fluid $[3 \bullet \bullet$. These guidelines cite the increased costs and unproven benefits in the rationale of this recommendation. It may be reasonable to utilize albumin to minimize the overall fluid administration in patients who likely are at high risk for fluid overload (e.g., patients with severe diastolic dysfunction or right-heart failure).

\section{Why Balanced Is Best}

First developed to treat dehydration brought about by the cholera outbreaks of the late 1800 s, salt solutions have been used for fluid resuscitation for over 100 years and remain the preferred first-line choice [15]. Crystalloids are available as either buffered solutions or unbuffered solutions. There are several buffered solutions available; however, the only unbuffered solution utilized in routine practice is saline. Buffered multi-electrolyte solutions vary in their composition but typically have a $\mathrm{pH}$, chloride concentration, and overall osmolality closer to human plasma than that of saline solutions.

Resuscitation with $0.9 \%$ saline has been associated, in a dose-dependent fashion, with the development of hyperchloremic metabolic acidosis, AKI, and potentially life-threatening end-organ dysfunction [15, 36, 37• 38••]. Despite these associations, over 200 million liters are used each year in the USA alone, and it remains a commonly preferred crystalloid solution [39]. 0.9\% saline is also a common diluent for many IV medications due to its inert chemical properties, often accumulating in an under-appreciated fashion as relatively large volumes of fluid administration [36].

The SPLIT trial randomized a mixed ICU patient population to receive either Plasma-Lyte (buffered) or $0.9 \%$ saline for maintenance or bolused IV fluids [40]. Patients in both treatment arms received an average of about $2 \mathrm{~L}$ of fluids. There was no difference in the rate of AKI or renal replacement therapy between the two groups. Although this study population was mixed, the majority of patients were post-operative. The majority of the postoperative patients were also elective procedures. The high percentage of elective post-operative patients may limit the generalizability of this study.

Two recent studies from Vanderbilt, SALT-ED and SMART, used a novel study design to allow for a very large randomized trial that included all patients admitted through either their Emergency Department (SALT-ED) or through any of their ICUs (SMART) [37•, 38••]. Based on the month, all patients within the system would receive either buffered solutions or normal saline. Lactated Ringer's was the most commonly used buffered solution 
in the study. The SALT-ED trial demonstrated no significant difference in a marker for short-term mortality, but normal saline was associated with an increased risk of AKI. The SMART trial also demonstrated an increased risk of adverse kidney events that could include death, renal replacement therapy, or persistent renal dysfunction for those patients treated with normal saline. In sub-group analysis, patients with sepsis were the most likely to be harmed using normal saline. In aggregate, these investigations provided the first conclusive prospective evidence for the utilization of balanced crystalloids in routine critical care practice.

\section{Conclusion}

Maintenance of adequate intravascular volume to promote cardiac output is a mainstay in the early management of septic shock. Vasodilation from increased nitric oxide production and a relative deficiency in vasopressin promotes vascular dilation and an increase in the unstressed volume. Further exacerbating the increased unstressed volume is the shedding of the EGL. Without the oncotic gradient across the EGL, the rate that water escapes from the intravascular space to the ECM increases, overwhelming the lymphatics and resulting in anasarca. The goals of fluid resuscitation are to restore the unstressed volume, optimize preload, and therefore optimize cardiac output and oxygen delivery. Current endpoints of resuscitation focus on administering fluid until the patient is no longer fluid responsive. At this point, vascular compliance and cardiac output are manipulated using vasopressors and inotropes, respectively. Colloids have not been demonstrated to be superior to crystalloids in the resuscitation of septic patients. Buffered crystalloids have been demonstrated to result in less kidney injury than $0.9 \%$ saline and should be the initial resuscitative fluid of choice. Future studies into therapeutic intervention targeting the EGL and optimizing the microcirculation, rather than simply the macrociruclation, are necessary and upcoming.

Authors' Contributions Conception and design: CSJ; structured literature review and interpretation: MWB and CSJ; drafting the manuscript for important intellectual content: MWB; revising the manuscript for important intellectual content and approval of the final version to be published: CSJ.

\section{Compliance with Ethical Standards}

Conflict of Interest Michael W. Best and Craig S. Jabaley declare they have no conflict of interest.

Human and Animal Rights and Informed Consent This article does not contain any studies with human or animal subjects performed by any of the authors.

\section{References}

Papers of particular interest, published recently, have been highlighted as:

- Of importance

- Of major importance

1. Singer M, Deutschman CS, Seymour CW, Shankar-Hari M, Annane D, Bauer M, et al. The third international consensus definitions for Sepsis and septic shock (Sepsis-3). JAMA. 2016;315(8): 801-10.

2. Rivers E, Nguyen B, Havstad S, Ressler J, Muzzin A, Knoblich B, et al. Early goal-directed therapy in the treatment of severe sepsis and septic shock. N Engl J Med. 2001;345(19):1368-77.

3.• Rhodes A, Evans LE, Alhazzani W, Levy MM, Antonelli M, Ferrer $\mathrm{R}$, et al. Surviving Sepsis Campaign: International Guidelines for Management of Sepsis and Septic Shock: 2016. Crit Care Med. 2017;45(3):486-552 The most recent guidelines from the Surviving Sepsis Campaign, often considered to set the standard of care for the management of patients with sepsis.

4.• Levy MM, Evans LE, Rhodes A. The Surviving Sepsis Campaign Bundle: 2018 update. Crit Care Med. 2018;46(6):997-1000 A new 1-h sepsis bundle is defined and recommended.

5. Woodcock TE, Woodcock TM. Revised Starling equation and the glycocalyx model of transvascular fluid exchange: an improved paradigm for prescribing intravenous fluid therapy. Br J Anaesth. 2012;108(3):384-94.

6. Nieuwdorp M, van Haeften TW, Gouverneur MCLG, Mooij HL, van Lieshout MHP, Levi M, et al. Loss of endothelial glycocalyx during acute hyperglycemia coincides with endothelial dysfunction and coagulation activation in vivo. Diabetes. 2006;55(2):480-6.

7. PE M. The physiology of volume resuscitation. Curr Anesthesiol Rep. 2014;4(4):353-9.

8. Becker BF, Jacob M, Leipert S, Salmon AHJ, Chappell D. Degradation of the endothelial glycocalyx in clinical settings: searching for the sheddases. Br J Clin Pharmacol. 2015;80(3): 389-402.

9. Marik P, Bellomo R. A rational approach to fluid therapy in sepsis. Br J Anaesth. 2016;116(3):339-49.

10. Johansson PI, Stensballe J, Ostrowski SR. Shock induced endotheliopathy (SHINE) in acute critical illness - a unifying pathophysiologic mechanism. Crit Care. 2017;21(1):25 A review of how endothelial changes during critical illness affect the disease process and observed pathophysiology.

11. Bentzer P, Griesdale DE, Boyd J, MacLean K, Sirounis D, Ayas NT. Will this hemodynamically unstable patient respond to a bolus of intravenous fluids? JAMA. 2016;316(12):1298-309 A review of various physiologic markers used to define fluid responsiveness with an evaluation of their sensitivities and specifities.

12. Lesur O, Delile E, Asfar P, Radermacher P. Hemodynamic support in the early phase of septic shock: a review of challenges and unanswered questions. Ann Intensive Care. 2018;8(1):102.

13. Jones AE, Shapiro NI, Trzeciak S, Arnold RC, Claremont HA, Kline JA, et al. Lactate clearance vs central venous oxygen saturation as goals of early sepsis therapy: a randomized clinical trial. JAMA. 2010;303(8):739-46.

14. Egal M, Erler NS, de Geus HRH, van Bommel J, Groeneveld ABJ. Targeting oliguria reversal in goal-directed hemodynamic management does not reduce renal dysfunction in perioperative and critically ill patients: a systematic review and meta-analysis. Anesth Analg. 2016;122(1):173-85.

15. MacDonald N, Pearse RM. Are we close to the ideal intravenous fluid? Br J Anaesth. 2017;119(suppl_1):i63-71. 
16. Investigators $\mathrm{A}$, et al. Goal-directed resuscitation for patients with early septic shock. N Engl J Med. 2014;371(16):1496-506.

17. Pro CI, et al. A randomized trial of protocol-based care for early septic shock. N Engl J Med. 2014;370(18):1683-93.

18. Mouncey PR, Osborn TM, Power GS, Harrison DA, Sadique MZ, Grieve RD, et al. Trial of early, goal-directed resuscitation for septic shock. N Engl J Med. 2015;372(14):1301-11.

19. Kuan WS, Ibrahim I, Leong BSH, Jain S, Lu Q, Cheung YB, et al. Emergency department management of sepsis patients: a randomized, goal-oriented, noninvasive sepsis trial. Ann Emerg Med. 2016;67(3):367-378 e3.

20. Andrews B, Semler MW, Muchemwa L, Kelly P, Lakhi S, Heimburger DC, et al. Effect of an early resuscitation protocol on in-hospital mortality among adults with sepsis and hypotension: a randomized clinical trial. JAMA. 2017;318(13):1233-40.

21. Investigators $\mathrm{P}$, et al. Early, goal-directed therapy for septic shock a patient-level meta-analysis. N Engl J Med. 2017;376(23):222334.

22. Rochwerg B, Alhazzani W, Sindi A, Heels-Ansdell D, Thabane L, Fox-Robichaud A, et al. Fluid resuscitation in sepsis: a systematic review and network meta-analysis. Ann Intern Med. 2014;161(5): 347-55.

23. Hernandez G, Teboul JL. Is the macrocirculation really dissociated from the microcirculation in septic shock? Intensive Care Med. 2016;42(10):1621-4 A review discussing when measures of the macrocirculation (e.g., blood pressure, stroke volume) is indicative or not indicative of microcirculation and the physiologic implications of these relationships.

24. Ince $\mathrm{C}$, Mik EG. Microcirculatory and mitochondrial hypoxia in sepsis, shock, and resuscitation. J Appl Physiol (1985). 2016;120(2):226-35.

25. Hahn RG, Lyons G. The half-life of infusion fluids: an educational review. Eur J Anaesthesiol. 2016;33(7):475-82.

26. Caironi P, Tognoni G, Masson S, Fumagalli R, Pesenti A, Romero $\mathrm{M}$, et al. Albumin replacement in patients with severe sepsis or septic shock. N Engl J Med. 2014;370(15):1412-21.

27. Finfer S, Bellomo R, Boyce N, French J, Myburgh J, Norton R, et al. A comparison of albumin and saline for fluid resuscitation in the intensive care unit. N Engl J Med. 2004;350(22):2247-56.

28. Annane D, Siami S, Jaber S, Martin C, Elatrous S, Declère AD, et al. Effects of fluid resuscitation with colloids vs crystalloids on mortality in critically ill patients presenting with hypovolemic shock: the CRISTAL randomized trial. JAMA. 2013;310(17): 1809-17.

29. Patel A, Laffan MA, Waheed U, Brett SJ. Randomised trials of human albumin for adults with sepsis: systematic review and meta-analysis with trial sequential analysis of all-cause mortality. BMJ. 2014;349:g4561.
30. Investigators SS, et al. Saline or albumin for fluid resuscitation in patients with traumatic brain injury. N Engl J Med. 2007;357(9): 874-84.

31. Brunkhorst FM, Engel C, Bloos F, Meier-Hellmann A, Ragaller M, Weiler $\mathrm{N}$, et al. Intensive insulin therapy and pentastarch resuscitation in severe sepsis. N Engl J Med. 2008;358(2):125-39.

32. Myburgh JA, Finfer S, Bellomo R, Billot L, Cass A, Gattas D, et al. Hydroxyethyl starch or saline for fluid resuscitation in intensive care. N Engl J Med. 2012;367(20):1901-11.

33. Perner A, Haase N, Guttormsen AB, Tenhunen J, Klemenzson G, Åneman A, et al. Hydroxyethyl starch 130/0.42 versus Ringer's acetate in severe sepsis. N Engl J Med. 2012;367(2):124-34.

34. Hebert PC, et al. A multicenter, randomized, controlled clinical trial of transfusion requirements in critical care. Transfusion Requirements in Critical Care Investigators, Canadian Critical Care Trials Group. N Engl J Med. 1999;340(6):409-17.

35. Holst LB, Haase N, Wetterslev J, Wernerman J, Guttormsen AB, Karlsson S, et al. Lower versus higher hemoglobin threshold for transfusion in septic shock. N Engl J Med. 2014;371(15):1381-91.

36. Magee CA, Bastin MLT, Laine ME, Bissell BD, Howington GT, Moran PR, et al. Insidious harm of medication diluents as a contributor to cumulative volume and hyperchloremia: a prospective, open-label, sequential period pilot study. Crit Care Med. 2018;46(8):1217-23.

37. Self WH, Semler MW, Wanderer JP, Wang L, Byrne DW, Collins $\mathrm{SP}$, et al. Balanced crystalloids versus saline in noncritically ill adults. N Engl J Med. 2018;378(9):819-28 A landmark study that demonstrated increased acute kidney injury associated with the use of $0.9 \%$ saline compared to balanced, buffered salt solutions in non-critically ill patients.

38.• Semler MW, Self WH, Wanderer JP, Ehrenfeld JM, Wang L, Byrne DW, et al. Balanced crystalloids versus saline in critically ill adults. N Engl J Med. 2018;378(9):829-39 A landmark study that demonstrated increased adverse kidney events associated with the use of $0.9 \%$ saline compared to balanced, buffered salt solutions in criticially ill patients.

39. Dalton C, Why did sterile salt water become the IV fluid of choice?, NPR. 2018: https://www.npr.org/sections/health-shots/2018/03/31/ 597666140/why-did-sterile-salt-water-become-the-iv-fluid-ofchoice. accessed 2/4/2018.

40. Young P, Bailey M, Beasley R, Henderson S, Mackle D, McArthur $C$, et al. Effect of a buffered crystalloid solution vs saline on acute kidney injury among patients in the intensive care unit: the SPLIT randomized clinical trial. JAMA. 2015;314(16):1701-10.

Publisher's Note Springer Nature remains neutral with regard to jurisdictional claims in published maps and institutional affiliations. 